communication earlier in the century. Interest in interstellar communication began within NASA at Ames Research Center in 1969. It was the subject of the Project Cyclops study in 1971, was given impetus by the Morrison workshops of 1975-76, and was funded for the development of equipment at the level of $\$ 1.5$ million from 1983-87. The strategy adopted was a microwave search at frequencies between one and ten Gigahertz. Ames was to carry out a targeted search of 1000 nearby solar type stars, while the Jet Propulsion Laboratory was to carry out an all-sky survey at less sensitivity. Observations were begun simultaneously on October 12,1992 at Arecibo, Puerto Rico for the targeted search and at the Deep Space Communications Complex at Goldstone in the Mohave Desert for the sky survey. By action of Congress, what was to be a ten year program of observations was cancelled in September of 1993.

Making use of taped interviews, archives and published sources, the NASA SETI History Project concentrates on four dimensions of this history: the political, technical, exploratory, and cultural. The political dimension includes internal and external consensus-building and funding aspects. The technical dimension concentrates on the design and construction of the MultiChannel Spectrum Analyzers, which will analyze millions of narrow channels simultaneously, as well as the interface of this equipment to the radio telescopes, and the subsequent detection software. The exploratory dimension explores how the project fits into the quest for humanity's place in the Universe. And the cultural dimension examines NASA's interest in the implications for society if an extraterrestrial civilization is successfully detected. This includes in the near term the post-detection protocols, as well as the long-term reaction of various elements of society.

With the termination of government funding, portions of the NASA project have been taken over by the SETI Institute, a non-profit institution that raises private funding. The NASA SETI History Project is in abeyance until further funding becomes available.

\title{
JOHN MELLISH AND THE CRATERS OF MARS
}

Michael Anderer, St. Charles, USA

This paper is an attempt to explore and evaluate the contribution of an American observer, John E. Mellish, to our understanding of Martian topography. Mellish (1886-1970) served as a research assistant at Yerkes Observatory in 1915-16 under Edwin Frost. Mellish had access to the 40" Clark refractor during this period which coincided with an aphelic opposition of Mars. He was subsequently claim that he observed "cracks and craters" near the Martian terminator in mid-november of 1915. A historical vignette of Mellish is undertaken as well as a technical analysis of the possibility that his claim had scientific validity. The author of the paper networked with Bradford Smithgh who serves on the Mars task force, committee on nomenclature who was able to advance the proposal that Mellish be recognized by having a crater named after him. The General Assembly of the IAU concurred and hence forth "Mellish" will be an officially designated crater. 
Discussion

D.H. DeVorkin : Just to comment that it might have been possible to see crater detail, Hale around 1908 (?) observed "faint interlocking curved filaments" on Mars with the 60" diaphragmed to $48^{\prime \prime}(?)$ in the region of what is now known as the crater EDOM. His observation was reported by Antoniadi in his Mars column, as well as by others. Hale was motivated to observe Mars as part of a site controversy with Percival Lowell and W.W. Campbell.

P. Moore : I have observed Mars with the 40 inches Yerkes refractor, and I am bound to say that I found it hard to believe that craters could be distinguished. Also, members may be interested to know that within two years the Rosse telescope at Birr Castle will again be working.

P. Brosche : Should one not generally ask for the reality of reported recognition of fine details by visual observers ? E.g. on the surface of the Moon by Philipp Fauth.

D.E. Osterbrock : I think your research on John Mellish at Yerkes Observatory was excellent. But I must say that I am sceptical that he observed craters on Mars visually in 1915. The fact that he did not report it in writing until 20 years later means his memory is all-important ; most memories tend to be very imperfect over such long times. Also, the fact that the reported observation was made three months from an aphelion opposition, but no one else reported a similar observation at closer (perihelion) oppositions at better sites, makes me sceptical. Of course no one could say that Mellish did not see the craters on Mars, but I would have to say that the verdict that he did see them is "not proved"

\section{A HISTORY OF AUSTRALIAN ASTRONOMY Raymond Haynes, Australia Telescope National Facility, Epping, Australia}

Overview. This study has been undertaken by Raymond Haynes, Roslynn Haynes, David Malin and Dick McGee. It provides the first detailed investigation of the development of astronomy in Australia. It looks both at the concepts of astronomy seen from the perspective of the Aboriginal peoples and, it provides the first complete review of the development of all branches of astronomy in Australia since 1788 and sets these into a social context of the day.The study is being published in a series of papers and in a book by Cambridge University Press (UK).

Areas of emphasis in the project. Australia contributes to research in fields as diverse as optical, radio, infra-red, cosmic-ray, x-ray, gamma-ray, and gravity-wave astronomy and plays a major role in international research contributing both scientists and instruments to the world community. This project is timely. Here I can only touch on some of the points addressed in the study. Aboriginal peoples have lived in Australia for over 50,000 years. Today less than 30 aboriginal languages survive out of the $120+$ that existed in 1788 . Their's was, and is, an oral culture rapidly being lost forever. An investigation of Aboriginal astronomy was overdue. Hopefully, our study helps rectify this. Many of the early white settlers were convicts. A strong trait that consequently developed and which has influenced the development of astronomy was the one of anti-authoritarianism, of rebelliousness to bureaucratic decision making. This trait showed up many times in our study. Another important factor was the one of isolation. Vast distances existed between early settlers in the country causing a 'make do' or 'fix it' mentality, which in turn led to a strongly innovative early community of astronomers. This still exists today. The early dependence on Britain for all things material was broken abruptly in 1851 with the discovery of gold in Australia. This in turn changed attitudes and relationships between the nascent astronomy community and Britain. The pursuit of astronomy provided a very clear sign of an awakening national 